\title{
Población indígena: inserción, desempeño y estrategias didácticas en los cursos computacionales de la Carrera de Administración de Oficinas, Universidad Nacional-Campus Sarapiquí
}

Indigenous populations: insertion, performance and didactic strategies in computing courses in the program for Office Management studies, Universidad Nacional - Sarapiquí Campus

\author{
Cristel Astorga Aguilar \\ Universidad Nacional, Costa Rica \\ cristel.astorga.aguilar@una.cr \\ Norieth Guillén Cordero \\ Universidad Nacional, Costa Rica \\ norieth.guillen.cordero@una.cr
}

Recibido: 30/04/2018 • Aceptado: 15/11/2018

\begin{abstract}
RESUMEN
El artículo muestra la percepción de los estudiantes indígenas del Campus Sarapiquí de la Universidad Nacional (UNA), sobre su inserción y desempeño en los cursos de aplicaciones computacionales de la carrera de Administración de Oficinas. El estudio realizado evidencia que la falta de conocimiento sobre el uso de la computadora, aunado a la resistencia de estas culturas a la tecnología, hacen que esta población ingrese rezagada a la universidad; por lo que se plantea cómo
\end{abstract}

1 Sección Regional Huetar Norte y Caribe, Campus Sarapiquí 
los antecedentes culturales y académicos de estos estudiantes, influyen en su rendimiento universitario. Además, se comparten estrategias didácticas propiciadas por las docentes de estos cursos, para facilitar el aprendizaje y la adaptación de esta población específica a la Universidad. Por último, se fundamenta la importancia de promover iniciativas que le faciliten al estudiante indígena una inserción exitosa y un rendimiento adecuado para garantizar su continuidad en la Universidad.

Palabras clave: TIC; identidad cultural; enseñanza superior; educación intercultural; población indígena.

\begin{abstract}
This article presents the perceptions of indigenous students in the Sarapiquí Campus of the Universidad Nacional (UNA) about their insertion and performance in computer applications courses in the program for Office Management studies. The study shows that the lack of knowledge about computer use, and resistance of these cultures to technology, causes these students to begin their university studies at a disadvantage. It is therefore important to understand how the cultural and academic backgrounds of these students influences their university performance. In addition, the didactic strategies promoted by teachers of these courses to facilitate the learning and adaptation process of this specific population to the University are discussed. Finally, the importance of promoting initiatives to facilitate successful insertion and adequate performance of indigenous students to guarantee their continuation at the University is justified.
\end{abstract}

Key words: ICT; cultural identity; higher education; intercultural education; indigenous population.

\title{
INTRODUCCIÓN
}

La Universidad Nacional, en virtud de su misión, en la que expresa su compromiso con la transformación democrática y progresiva de las comunidades hacia planos superiores de bienestar, preferentemente con aquellas menos favorecidas o en el riesgo de exclusión y, además, de su visión, en la que se refleja su responsabilidad con el desarrollo humano integral, incluyente, equitativo y en respeto de los derechos humanos, ha realizado 
múltiples esfuerzos que han favorecido, de manera directa o indirecta, a los pueblos indígenas (CONARE, 2013). Además, en conjunto con el Consejo Nacional de Rectores (CONARE), la Universidad Estatal a Distancia (UNED), la Universidad de Costa Rica (UCR) y el Instituto Tecnológico Costarricense (TEC), ha sido parte de una iniciativa que busca específicamente la inclusión, permanencia y éxito de los estudiantes indígenas en la Educación Superior, con el fin de garantizar el cumplimiento de los derechos de los pueblos indígenas.

En el Proyecto de Mejora de la Educación Superior, Marco de Planificación para Pueblos Indígenas (MPPI) (CONARE, 2013), se recomienda a las universidades integrar actividades con los pueblos indígenas:

...contemplen formas articuladas e interuniversitarias para complementar esfuerzos y maximizar recursos. Esto se debería tomar en cuenta especialmente en la utilización de sedes e instalaciones regionales para ampliar la oferta académica en áreas geográficamente más accesibles para las poblaciones indígenas, tomando en cuenta las necesidades y demandas académicas de ellos (p. 13).

Es así como, después de diversos esfuerzos, en 2015 la Sección Regional Huetar Norte y Caribe, Campus Sarapiquí de la Universidad Nacional, recibe por primera vez población estudiantil proveniente de territorios indígenas. Esto amparado en los principios humanista y de inclusión de la UNA con los sectores menos favorecidos por razones económicas, culturales o por discapacidad.

Asimismo, como producto del trabajo con los pueblos originarios, desde diferentes proyectos de extensión e investigación, así como la incorporación casi sistemática de estudiantes de diferentes etnias indígenas, nace la iniciativa de declarar a la Sección Regional Huetar Norte y Caribe como Campus de Interés Intercultural, hecho que se da el 31 de mayo del 2018 con el acuerdo UNA- SCU-ACUE-1040-2018 de la gaceta ordinaria \#9. Dicha declaratoria evidencia el compromiso institucional que se tiene con la construcción y el fortalecimiento de la docencia a través de procesos interculturales. 
Una de las carreras que cuenta con población indígena es la de Administración de Oficinas que, como parte de su plan de estudios, tiene establecido el abordaje de cursos de computación que permitan desarrollar las competencias necesarias para el manejo de la computadora en cuanto a Windows, Microsoft Office, administración de documentos electrónicos. Asimismo, se implementa el uso de aplicaciones tecnológicas en línea que faciliten y coadyuven en el trabajo de oficina; además de adquirir los conocimientos, las destrezas, las habilidades y las aptitudes para producir documentos que se requieren en las empresas.

En el transcurso de cuatro años a partir del 2015, las investigadoras determinan que existe carencia de formación básica por parte del grupo estudiantil indígena que, en su mayoría, presenta resistencia y dificultad para la asimilación de herramientas computacionales. Dichas dificultades se evidencian también en el porcentaje de reprobación en los cursos de computación de la carrera.

En razón de lo anterior, el objetivo de esta investigación es conocer la percepción de los estudiantes indígenas del Campus Sarapiquí de la Universidad Nacional (UNA), sobre su inserción y desempeño en los cursos de aplicaciones computacionales de la carrera de Administración de Oficinas. El artículo, en primera instancia, realiza una identificación de las poblaciones indígenas que existen en Costa Rica. Seguidamente, se analiza la importancia de que el estudiante indígena que cursa la carrera en cuestión, aprenda aplicaciones computacionales. Posteriormente, se hace referencia a los antecedentes culturales y académicos de la población en estudio, así como a la interculturalidad y estrategias didácticas utilizadas con los estudiantes indígenas. Asimismo, se detalla la metodología utilizada para efectuar la presente investigación y los resultados que incluyen percepción del estudiante indígena sobre su inserción y desempeño en los cursos de aplicaciones computacionales. Finalmente, se presenta la discusión, las conclusiones arrojadas a partir del proceso y las recomendaciones.

\section{Poblaciones indígenas de Costa Rica}

En relación con los grupos étnicos del país, el décimo octavo informe Estado de la Nación en Desarrollo Humano Sostenible estable lo siguiente: 
En Costa Rica el sistema reconoce -por medio del Decreto $\mathrm{N}^{\circ}$ 20645 de 1991- ocho pueblos indígenas - a los que le denomina "grupos étnicos" (Bribrí, Cabecar, Guaymí -que se reivindica como Ngöbe-, Brunca, Térraba, Huetar-que el Decreto también denomina "Pacacua"; Maleku - que el Decreto llama "Guatuso"- y Chorotega) que viven en uno o más de los 24 territorios indígenas reconocidos (Programa Estado de la Nación, 2012, p.7).

Por su parte, el Cuarto Informe del Estado de la Educación, refiere:

Grupo étnico Cabécar: Territorios indígenas Alto Chirripó, Bajo Chirripó, Tayní, Teire, Talamanca Cabécar, Ujarrás, Nairi Awari, China Kichá. Grupo étnico Bribri: Territorios indígenas Talamanca Bribrí, Cocles (Kekoldi), Salitre, Cabagra. Grupo étnico Brunca (boruca): Territorios indígenas Boruca, Curré. Grupo étnico Térraba (teribe): Territorio indígena Térraba. Grupo étnico Guaymí (ngöbe): Territorio indígena Guaymí de Coto Brus, Abrojo Montezuma, Conte Burica, Gaymi de Osa, Altos de San Antonio. Grupo étnico Huetares: Territorios indígenas Quitirrizí, Zapatón. Grupo étnico Guatuso (Malekus): Territorio indígena Guatuso. Grupo étnico Chorotega: Territorio indígena Matambú (Borge, 2012, p.11).

Es importante referenciar que los estudiantes indígenas de la Sección Regional Huetar Norte y Caribe, Campus Sarapiquí de la Universidad Nacional, que estudian la carrera de Administración de Oficinas, pertenecen a los grupos étnicos Bribri, Cabécar y Maleku.

Salazar (2006), realiza una ubicación general de los territorios. En cuanto a los cabécares, indica lo siguiente:

Habitan el territorio boscoso más grande del país, en la selva húmeda lluviosa y los sitios escarpados del macizo montañoso: Chirripó, Telire, Alto Chirripó, Nairí, Paso Marcos, a orillas del río Pacuare, Quetzal, Veré, Corina, Valle Escondido, Pozo Azul, Barbillas, San Pedro de Río Coén, El Dos de Moravia, Alto Celia, VaIle La Estrella, Mojocito, Shiroles, Suretka, Gavilán Canta, Awarí y Orochico (p.15). 
Sobre los Bribis, señala que este pueblo constituye la mayor población indígena del país y que "habita en varios asentamientos en las llanuras de Baja Talamanca a orillas de los ríos afluentes del Sixaola y en el macizo montañoso, en particular en la vertiente atlántica" (Salazar, 2006, p.16).

Por su parte, Salazar (2006), menciona de los Guatusos que "son el grupo más pequeño y se autodenominan Maleku, que también es el nombre del idioma que hablan" (p. 17). Además, que "habitan en la región del Atlántico Norte Central del país, conocida como Llanuras de Guatuso" (p. 18).

\section{Importancia del aprendizaje de aplicaciones computacionales: TIC y población indígena}

La carrera de Administración de Oficinas de la Universidad Nacional busca que los estudiantes estén académicamente preparados para asumir los retos tecnológicos de la oficina moderna y las exigencias del mercado laboral actual, donde la tecnología forma parte imprescindible de las tareas administrativas. Por ello, para ser un ejecutivo contemporáneo, es necesario conocer y utilizar adecuadamente los diferentes sistemas digitalizados de trabajo, equipo de oficina, servicios de internet y demás aplicaciones computacionales que facilitan el quehacer de la empresa.

En ese sentido, es prioritario que los programas de estudio de las carreras relacionadas con la gestión de oficinas y empresas, tengan incluida la tecnología como eje temático, de manera que los estudiantes aprendan y practiquen todas las herramientas que les serán de utilidad para desempeñarse exitosamente en su futuro trabajo. De no ser así, se estarían graduando desactualizados y tecnológicamente incompetentes, lo que puede dificultar su inserción en el mercado laboral y los obligaría, tarde o temprano, a incurrir en gastos económicos y tiempo de capacitación adicional para poder actualizarse y encontrar un trabajo.

La tecnología también ha influido grandemente en la globalización de las economías, afectando directamente la manera como las empresas realizan negocios. Según Barragán y Vela (2015):

Los tomadores de decisión empresarial tienen que evaluar el impacto que tiene la tecnología en su corporativo, porque hoy en día es el centro de cualquier iniciativa. Las TICs son tan importantes 
en las empresas e industrias que en muchos escenarios son factor de éxito y nivel de competitividad alto (p.19).

Esta necesidad de las empresas, de ajustarse a las exigencias del mercado en el que compiten, hace que sea inminente la incorporación de las tecnologías de la información y comunicación (TIC) en sus operaciones.

Seas Tencio (2016) define las tecnologías de la información y la comunicación (TIC) como "los dispositivos o instrumentos, métodos y técnicas para el logro, el tratamiento, el almacenamiento y la transmisión de información" (p. 290). Estas innovadoras técnicas para la gestión de la información han revolucionado la manera en que se realizan los procesos en las diferentes oficinas, han mejorado la sistematización de los datos, el procesamiento de la información, los canales de comunicación, el control interno. También, han disminuido el tiempo para realizar los trámites, permiten que no haya fronteras para realizar negocios, aumenta la exposición de la empresa vía internet, lo que facilita el incremento de las ventas, entre otras grandes ventajas. Es por esto que, actualmente, tanto las grandes industrias, como las micro, pequeñas y medianas empresas apuestan al uso de la tecnología para mejorar su quehacer.

En relación con las TIC en la gestión administrativa, Saavedra (2017) indica que

Actualmente ninguna empresa puede funcionar sin las TIC, es a través de ellas, que todo se resuelve con mayor facilidad. El mundo está informatizado, la informática tal vez sea el área que más influenció el curso del siglo XX. Si hoy vivimos en la Era de la Información, esto se debe al avance tecnológico en la transmisión de datos y a las nuevas facilidades de comunicación, ambos impensables sin la evolución de las computadoras y dispositivos (p. 111).

Sin embargo, para hacer uso correcto de las tecnologías en las empresas, es necesario contar con personal capacitado que pueda darle un uso adecuado y eficiente a los recursos. De ahí la importancia de que las personas desarrollen la competencia de manejar los equipos tecnológicos y aplicaciones computacionales con que cuentan las empresas para su crecimiento y desarrollo económico. 
Se debe recordar que "los beneficios de adquirir tecnologías de información se dan cuando se obtiene conciencia de que las ventajas competitivas y la sostenibilidad de los negocios, se aseguran con el adecuado uso de la tecnología" (Prada Ospina, 2016, p. 112). Por lo que el papel del trabajador es fundamental como ente facilitador de la supervivencia tecnológica de la organización.

Con el aumento de la exposición a la tecnología que viven los seres humanos cotidianamente, ha sido necesario asumir un proceso prácticamente diario de actualización digital, con el fin de hacer uso de los recursos tecnológicos que están en un proceso constante de cambio y mejora.

Si bien es cierto las herramientas tecnológicas pueden representar riesgos para la sociedad, también es incuestionable que presentan una serie de ventajas que permiten a las personas ser parte y estar al tanto de lo que acontece en el mundo. Es posible comunicarse diariamente por medio de teléfonos celulares y sus diferentes aplicaciones, recibir noticias en tiempo real desde cualquier lugar del mundo, realizar compras en línea, hacer actividades de ocio, ofrecer servicios en línea, almacenar datos ilimitados, tener acceso a cualquier tipo de información disponible en la web desde prácticamente cualquier lugar, hacer aportes sobre temáticas personales, sociales, políticas, académicas y de cualquier otra materia libremente mediante redes sociales, blogs, grupos de discusión, foros, videos, entre otros; facilidades que antiguamente eran impensables.

La introducción de estas y otras nuevas tecnologías en la estructura social han planteado lo que hoy se llama la revolución científico-tecnológica. Revolución generadora de grandes cambios socioculturales que abren el camino de una nueva sociedad que está surgiendo con diversos nombres, entre ellos: sociedad del conocimiento (Gay, 2008).

Actualmente, todos los individuos, sin importar su edad, género, grupo étnico, profesión, nacionalidad, religión, creencias o costumbres, pueden beneficiarse de ser digitalmente competentes, por lo que hay que asegurar el aprendizaje de habilidades tecnológicas en la escuela, como la etapa de la enseñanza obligatoria. Es necesario que se aprenda utilizando los ordenadores y demás dispositivos digitales; por eso, no se puede tener miedo a que los alumnos estén mucho (entre un $30 \%$ y un $50 \%$ del tiempo 
de escuela) conectados a Internet, porque deben ser educados como personas morales y usuarias inteligentes de Internet (García, 2016).

Generalmente, los jóvenes están acostumbrados a trabajar con tecnología, es parte de su vida y la aplican para todo. Prensky (2011) menciona lo siguiente:

Más y más gente joven ha mejorado profunda y permanentemente en cuanto al uso de la tecnología, conectando con sus iguales y el mundo como ninguna generación anterior lo había hecho. Ríos de información les llegan las veinticuatro horas del día de los siete días de la semana. Cada vez en mayor medida lo que quieren y necesitan está disponible en su bolsillo bajo demanda. (p. 12).

Es obligación de la educación universitaria seguir desarrollando las competencias necesarias para que el educando pueda estar a la vanguardia de los cambios tecnológicos a los que se enfrenta el mercado laboral.

\section{Antecedentes culturales y académicos de la población en estudio}

La corriente tecnológica se evidencia día con día en los diferentes ambientes en los que se desenvuelven las personas; tanto en el plano familiar, como social y, por supuesto, el sector educativo se encuentra altamente influenciado. Sin embargo, hay estudiantes que por diversas razones no tienen la misma exposición a la tecnología; tal es el caso de la población que atañe a esta investigación: los estudiantes de origen indígena. Algunos de los factores que dificultan su relación con la tecnología son: posibilidades económicas limitantes para adquirir computadoras, teléfonos y otros dispositivos, dificultad de sus centros educativos para obtener recursos tecnológicos, falta de acceso a internet por su lugar de residencia, entre otras.

Según Fallas Monge y Trejos Trejos (2015), la brecha digital es "aquella separación que existe entre las personas, comunidades o países que utilizan las Tecnologías de la Información como una parte rutinaria de su vida diaria y aquellas que no tienen acceso y aunque las tengan, no saben cómo utilizarlas" (p. 89). Además, hacen mención que esta puede ser definida en términos de desigualdad de posibilidades que existen para acceder a la información, al conocimiento y a la educación, mediante el uso de 
la tecnología, situación que se contextualiza a la población indígena que presenta problemáticas para el uso de la tecnología.

Hernández y Calcagno (2003) indican que el imparable desarrollo tecnológico crea una brecha digital para los pueblos originarios que los margina de las tecnologías de información y comunicación, lo que los limita para incorporarse laboralmente. Para la mayoría de los indígenas, las TIC son bienes de la sociedad global inaccesibles, destinados únicamente para sectores privilegiados, lo cual los distancia técnica y culturalmente.

Para la población indígena, el acceso a la tecnología y el manejo de los diferentes equipos y aplicaciones tecnológicas demanda el doble de esfuerzos, al no contar con los recursos tanto económicos, como educativos a temprana edad, para poder encaminarse hacia el desarrollo de competencias tecnológicas. Esto no solo es un tema de rezago o falta de acceso; las comunidades indígenas tienen resistencia al desarrollo social, tecnológico y hasta económico por la dinámica interna de estas sociedades, sus comunidades y sus liderazgos. Se pueden mencionar, como ejemplos de lo anterior, la inequidad de género que viven, situación que limita la evolución de la mujer y la des-ruralización de los miembros más jóvenes, que genera rivalidades entre los que se fueron y los que se quedaron (Hernández y Calcagno, 2003).

Mientras que algunas poblaciones indígenas han visto en la tecnología una oportunidad para trascender, también hay sectores que "levantan voces que denuncian a las nuevas tecnologías como una forma de injerencia de la sociedad nacional, que busca sumar a las comunidades al consumo informático pautado desde intereses ajenos" (Hernández y Calcagno, 2003, p. 122). Las características expuestas anteriormente, aunadas al proceso de adaptación que deben vivir los estudiantes al ingresar a la universidad, influyen significativamente en la inserción exitosa dentro los cursos de aplicaciones computacionales.

Czarny (2007) plantea la escolaridad como:

Un acto migrante, en tanto que implica, para todos los sujetos (no sólo para las comunidades indígenas), el pasaje a otras prácticas de socialización y constitución de subjetividades -de tensión, confrontación, negociación, cercanía, distancia, etcétera-, 
diferenciadas de las prácticas de socialización iniciales o primarias, que para las comunidades indígenas en ese acto se expresan, en mayor o menor grado, los desencuentros socioculturales que ha producido el acceso a la escolaridad (p. 944).

Para las personas indígenas, la transición y adaptación que conlleva el ingreso a la Universidad, como lo indican Luján y Torres (2014) en su ponencia: "Jóvenes indígenas y educación superior. Experiencias en el acceso y permanencia en la Universidad", significa una multiplicidad de cambios en sus estructuras habituales, rupturas en el plano académico y aquellas nuevas experimentadas en la universidad. Asimismo, el movimiento migratorio que estos estudiantes realizan, genera distintos procesos, a diferencia de los estudiantes que residen en las ciudades donde se ubican las unidades académicas, los condiciona a adquirir otros tipos de aprendizajes que van más allá de lo académico e institucional.

Dicha dificultad ha sido también evidenciada por CONARE (2013), cuando indica entre las conclusiones del Proyecto de Mejora de la Educación Superior, Marco de Planificación para Pueblos Indígenas (MPPI), que entre las barreras que afectan el acceso de las poblaciones indígenas a la educación superior destacan:

...la falta de acceso y calidad de la formación secundaria, el aislamiento geográfico de los territorios de los centros universitarios, la percepción sobre el potencial desarraigo a su comunidad e identidad cultural, la situación económica precaria y el costo de oportunidad que conlleva los estudios universitarios; el pobre acceso a información orientadora clave sobre requisitos y oportunidades universitarias, la falta de adecuación en los procesos de inscripción, admisión y matricula. Así mismo, una fuerte barrera la constituye la tecnología ya que implica una serie de trámites en línea (becas, matrícula, entre otros) que representan complejidad para un estudiante indígena (p. 12).

La presencia de los factores anteriores en la población indígena de la UNA, Campus Sarapiquí, apunta a la necesidad de implementar estrategias que faciliten al estudiante indígena el proceso de enseñanza y aprendizaje de tecnologías. Más aun, tomando en cuenta que con muchos de ellos lo que se requiere es iniciar desde cero con un proceso de alfabetización digital 
"alfabetización basada en destrezas que consiste en instruir en conceptos y procedimientos básicos de las TIC, aprender a leer y escribir con el nuevo lenguaje propio de las tecnologías, comunicarse con la tecnología con el objeto de conocer los retos y oportunidades así como las amenazas y límites que aportan su uso" (Solarte, Urbano, y Triviño, 2007, p. 67); pues esta temática no fue un contenido prioritario en sus colegios.

En algunas ocasiones, el acceso a la educación para este tipo de poblaciones a nivel superior es una meta que se ve inalcanzable por factores económicos, sociales y culturales. Sin embargo, Paladino (2009) menciona que cuando se investiga sobre las causas del escaso porcentaje de jóvenes indígenas que cursan estudios de nivel superior, se confrontan problemáticas no solo económicas, de distancia espacial, de barreras lingüísticas, sino también, procesos de autoexclusión por considerar este tipo de educación inaccesible, relativo a un grupo social y a una cultura y forma de conocimiento ajenas.

\section{Interculturalidad y estrategias didácticas utilizadas con estudiantes indígenas}

Con el fin de facilitar el acceso, la pertinencia y la permanencia de los estudiantes indígenas en la universidad, es necesario adoptar un enfoque intercultural. Este se entiende como el reconocimiento de que todos los estudiantes vienen de contextos diferentes, en los que sus actividades, creencias, costumbres y tradiciones, no son las mismas, por lo que es importante reconocer y aceptar esas diferencias y tomarlas en cuenta para propiciar la igualdad de condiciones dentro de la clase.

De acuerdo con Cabrera y Gallardo (2013):

La educación intercultural constituye un enfoque educativo basado en el respeto y la valoración de la diversidad de la cultura de origen, proponiendo modelos de intervención, formales e informales, holísticos, integrados, configuradores de todas las dimensiones del proceso educativo para lograr la igualdad de oportunidades y resultados. (p. 4).

El respeto hacia la diversidad es trascendental por parte de los todos los involucrados en el proceso educativo; sin embargo, en muchas ocasiones 
no se aborda de manera integral, lo que evidencia resultados parciales. Según Sánchez et al. (2009):

Una pedagogía intercultural adecuada puede lograr la reversión de las desigualdades existentes entre las zonas urbanas y rurales, si es capaz de generar oportunidades para el desarrollo de competencias para todos los educandos de una forma integral, y reconociendo la particularidad personal y el respeto hacia los otros. En otras palabras, se necesita de una política educativa en donde la interculturalidad sea una opción que sirva para eliminar aquellas tendencias de invisibilizar la diversidad cultural en determinado territorio (p. 2).

A partir del 2015, el proceso educativo en la carrera Administración de Oficinas de la UNA, Campus Sarapiquí se enriqueció al poder brindar una formación intercultural. También, se evidenció que los índices de reprobación de cursos en el área de tecnología aumentaron, ya que un porcentaje de jóvenes indígenas ha reprobado uno o más cursos de la carrera.

Amado, García, Brito, Sánchez y Sagaste (2014), sobre la reprobación estudiantil, mencionan que su disminución es tarea de todos los actores involucrados en el proceso, generando cambios en la forma en que los estudiantes visualizan su estadía en las instituciones de educación superior, la forma en que los docentes realizan su labor y una intervención inmediata de las autoridades educativas, mediante la aplicación de mecanismos de control e instancias de toma de decisiones. Asimismo, citan a Espinoza García, incluyendo entre las causas de reprobación escolar la "...desadaptación al medio por el origen sociocultural del que provienen...” (p. 236).

El aprendizaje debe ser un proceso que se construye por medio de la interacción y la reflexión, generando ambientes propicios y herramientas cognitivas que ayuden al discente a diseñar e implementar soluciones ante posibles interrogantes. Seas Tencio (2016), menciona lo siguiente respecto del aprendizaje:

... este puede ocurrir con o sin procesos de enseñanza formales; puede suceder de forma natural y a lo largo de toda la vida, por la experiencia personal individual o por la interacción con otros; en la escuela, pero también en la sociedad en general, puede 
adquirirse de los docentes, de cualquier otra persona, en forma individual y grupal. Se aprenden contenidos, pero también a pensar, a relacionarse con otros; así como las emociones y los sentimientos, los valores y la identidad cultural (p. 34).

El proceso puede darse como parte de un sistema educativo formal, pero también de manera informal: las personas aprenden de manera empírica desde el hogar y la comunidad, máxime cuando se trata de tecnología y generaciones jóvenes. Por otro lado, en los procesos educativos formales, resalta la figura del facilitador, elemento sumamente importante para lograr los objetivos y metas planteadas; en este caso en particular, el desarrollo de habilidades y destrezas para la manipulación correcta de aplicaciones computacionales de mayor uso en las oficinas.

Es importante que el papel del docente se enmarque en una actitud de colaboración y también de aprendizaje, pues es necesario aprender sobre el contexto socio-cultural de la población indígena para trabajar las lecciones en respeto de sus necesidades. Además, para dar paso al reconocimiento de aportes y diferencias de otras culturas, permitiendo que la educación superior costarricense se rija por principios de interculturalidad y facilitando, así, la permanencia de los estudiantes indígenas en la Universidad.

Por su parte, González Oviedo (2009), hace referencia a que la interculturalidad no solo da cuenta de las relaciones entre distintos grupos culturales, sino que implica una visión de futuro en la medida en que pretende que dichas relaciones sean simétricas, incluyentes y no mediatizadas por discriminaciones y desigualdades de ningún tipo. Así, la interculturalidad se vincula con el desarrollo humano, en lo que la educación tiene un papel fundamental. Sin embargo, tradicionalmente se piensa que todos tienen las mismas necesidades educativas, las mismas expectativas sobre la educación y la misma forma de aprender y de interiorizar el conocimiento.

El docente debe tener claro que las actitudes, deseos y necesidades cambian de una cultura a otra y, por ende, a partir de diagnósticos se debe trabajar en la búsqueda de técnicas y recursos didácticos que permitan que todos asimilen los nuevos conocimientos. Este proceso es trascendental para el éxito en el ámbito educativo, ya que al no lograr que el estudiante se encuentre motivado en la clase, se fomentará la deserción, el menor aprovechamiento de los contenidos formativos y la escasa correspondencia de 
lo que se enseña con el conocimiento y las habilidades requeridas para la vida cotidiana y su posterior desempeño profesional.

González Oviedo (2009), sobre la diversidad cultural y la interculturalidad, cita lo siguiente:

No se puede establecer un modelo educativo que no reconozca la diversidad cultural y que no incluya la interculturalidad como eje transversal. Una educación de calidad que responda a las exigencias formativas de la interculturalidad, debe adecuar tanto los contenidos, como los métodos de enseñanza y aprendizaje, de manera que estos contemplen la construcción simbólica de la realidad y la visión de desarrollo (p. 250).

La construcción a partir de la diversidad, valorando y respetando las diferencias, requiere convivencia que permita reconocer mismos derechos y misma dignidad, así como comprensión de las distintas culturas. Dentro de la metodología del proceso de enseñanza se deben tomar en consideración los valores, saberes, conocimientos, lenguas y otras expresiones culturales con la finalidad de facilitar los espacios idóneos para el aprendizaje. Viveros-Márquez y Moreno-Olivos (2014) mencionan que "la pedagogía intercultural considera el contexto social y cultural del sujeto y la cultura juega un papel muy importante y central en el proceso de aprendizaje, y se concibe como algo dinámico, cambiante y adaptativo" (p. 60).

El desarrollar procesos enfocados desde la interculturalidad implica interacción, intercambio e interrelación entre culturas. No se trata de homogenizar, ya que de esta forma se estaría excluyendo; la equidad, calidad, pertinencia y construcción en conjunto deben estar presentes en la estrategia educativa. Por lo tanto, se debe contextualizar la educación a las distintas realidades socioculturales para que responda a las necesidades de cada persona; para ello, la selección de los recursos educativos y medios didácticos adecuados son significativos para el éxito del proceso.

Fallas Monge y Trejos Trejos (2015) definen recurso educativo a "cualquier material que se utilice con una finalidad didáctica o para facilitar el desarrollo de las actividades de formación" (p. 102). Asimismo, precisan que los medios elaborados, específicamente, para facilitar el proceso de enseñanza y aprendizaje son medios didácticos. 
Particularmente en los cursos de tecnología de la carrera de Administración de Oficinas, las académicas investigadoras han utilizado varias técnicas didácticas con el fin de cumplir con los objetivos educativos. Considerando las metas de cada curso algunas se han adaptado, se han mejorado y se han evaluado constantemente. Como parte de su aplicación, es necesaria la guía constante y el seguimiento oportuno por parte del facilitador, su validación y la disposición de los recursos requeridos; además, para asegurar el éxito de la técnica, se deben fomentar procesos comunicativos, participativos y de mediación por parte del docente.

Seas Tencio (2016) menciona que es importante trabajar bajo una práctica docente constructiva y colaborativa, respetuosa de las diferencias individuales y del contexto, donde la labor docente emprenda la búsqueda de actividades y estrategias adecuadas, a fin de alcanzar aprendizajes significativos. Bajo esa premisa, en los cursos de tecnología se han utilizado las siguientes estrategias didácticas con población indígena:

- Visitas y experiencias directas con propósito: los estudiantes asisten a las oficinas con el fin de evidenciar las prácticas administrativas y desarrollar conductas en espacios reales. Por ejemplo, en la elaboración de tipos documentales: cartas, memorandos, circulares, constancias, certificaciones, curriculum vitae, actas e informes.

- Discusiones guiadas: para la generación de interés y la activación o identificación de conocimientos previos.

- Utilización de medios audiovisuales como imágenes, audios y videos como complemento de los contenidos desarrollados en los cursos, tanto por las docentes como por los estudiantes cuando se realizan exposiciones.

- $\quad$ Programas de aplicaciones informáticas: procesadores de texto, hojas electrónicas, programas de presentaciones y bases de datos.

- Juegos educativos: actividades lúdicas que permitan la realimentación de contenidos.

- $\quad$ Programas de ejercitación: programas con ejercicios para el desarrollo de la destreza mecanográfica. 
- Tutoriales digitales: con la correcta manipulación de herramientas o aplicaciones informáticas.

- Internet: para la búsqueda de información y el refuerzo de los contenidos.

- Correo electrónico: para el intercambio de información entre profesor y estudiante y viceversa, así como para el trabajo colaborativo.

- Foros de discusión: aplicación tecnológica que permite el análisis de temáticas de manera colaborativa.

- Exposición verbal: para propiciar aprendizajes significativos.

- Conversaciones electrónicas o chats: con la finalidad de reforzar el desarrollo de la destreza mecanográfica.

- $\quad$ Aulas virtuales: uso de plataformas diseñadas para facilitar la comunicación no presencial y la evaluación de los aprendizajes.

- $\quad$ Prácticas guiadas: para propiciar aprendizajes significativos, reconocer y reflexionar sobre errores cometidos.

- Trabajo en grupos: para facilitar el aprendizaje colaborativo.

- Presentaciones en programas informáticos desarrollados para este fin: para poner en práctica conocimientos adquiridos y la investigación de herramientas tecnológicas.

- Trabajo por proyectos: donde se fomenta la investigación y el trabajo colaborativo, así como sus habilidades de comunicación oral y escrita.

Asociado a la aplicación de las técnicas didácticas anteriores con población indígena, se deben aplicar diagnósticos con el fin de determinar conocimientos previos, así como temáticas que se deben reforzar. La comunicación es sumamente importante, ya que por sus personalidades no expresan sus necesidades, por lo que el docente debe estar en una constante búsqueda de estrategias que le permitan monitorear si el objetivo de 
la clase se está logrando. Las relaciones interpersonales se deben abordar para que poco a poco la comunicación sea más fluida.

Otra estrategia que se utiliza con frecuencia en el Campus Sarapiquí es el desarrollo de tutorías en las materias que presentan problemas de reprobación o dificultad, espacio que está disponible para todos los estudiantes matriculados en el curso, por lo tanto, este es un espacio de refuerzo que también puede utilizar la población indígena.

Asimismo, la experiencia obtenida por las investigadoras permite indicar que es necesario reforzar los canales de comunicación que permitan identificar esas necesidades, ya que muchas veces el estudiante indígena no se comunica como el profesor está acostumbrado tradicionalmente; no expresan sus limitantes y no indican en cuáles contenidos hay dudas. Por lo tanto, el reto no es solo para los discentes, sino que también incumbe al profesor que desarrolla los cursos de tecnología, principalmente si nunca ha trabajado con población indígena.

El papel del docente también implica sensibilizar, contribuir a la paz y la convivencia humana, es trabajar para que todos se sientan con los mismos derechos y deberes y asuman un rol activo en el proceso de aprendizaje y aporte con la sociedad. Trabajar en la aprobación y la aceptación de la cultura de los otros sin renunciar a la propia.

Por su parte, Mandiola (s.f.), en un artículo para la UNESCO con respecto a educación e interculturalidad cita lo siguiente:

Una propuesta de educación e interculturalidad plantea un diálogo entre iguales y en igualdad de condiciones, el encuentro entre personas y culturas, el intercambio de saberes, hacia una educación intercultural, inclusiva y diversa. La riqueza de los saberes y conocimientos de cada cultura es uno de los objetivos fundamentales de la educación contemporánea. Es necesario que este objetivo se concrete no únicamente en contenidos o materias, sino en los mismos principios, enfoques y programas nacionales educativos, así como en la misma institucionalidad educativa.

La Universidad Nacional, a través de sus principios, tiene trazado el camino hacia el conocimiento transformador, el humanismo, la inclusión así 
como en su valores por medio del compromiso social, la equidad, la participación democrática y el respeto; elementos que se entrelazan en sus fines reflejados en el diálogo de saberes, la identidad y el compromiso, la formación integral y el pensamiento crítico. Está en su quehacer, enmarcada la ruta hacia el fortalecimiento de la identidad, las relaciones democráticas y la construcción de modelos donde formen parte el intercambio de saberes y el desarrollo humano.

En la Sección Regional Huetar Norte y Caribe, la población indígena dirige procesos y actividades estudiantiles en representación de toda la UNA. Esto evidencia el empoderamiento que tienen como representantes y líderes estudiantiles del Campus, así como de los pueblos indígenas que representan.

\section{METODOLOGÍA}

La experiencia obtenida a partir del 2015 al recibir población indígena en la carrera de Administración de Oficinas permitió evidenciar problemáticas en el desempeño y aprobación de los cursos de computación. En razón de lo anterior, se realiza un estudio de investigación bajo el enfoque cuantitativo porque, "parte de una idea, que va acotándose y, una vez delimitada, se derivan objetivos y preguntas de investigación, se revisa la literatura y se construye un marco o una perspectiva teórica" (Hernández, Fernández, y Baptista, 2010, p. 4). Asimismo, la recolección de los datos se fundamenta en la medición.

La investigación se desarrolla en la Sección Regional Huetar Norte y Caribe de la Universidad Nacional, en Sarapiquí de Heredia, Costa Rica, a estudiantes regulares pertenecientes a algún territorio indígena y que cursan la carrera de Administración de Oficinas. Con respecto a la población en estudio, se considera a la totalidad de estudiantes de origen indígena del bachillerato en Administración de Oficinas del Campus Sarapiquí de la Universidad Nacional, por lo que no fue necesario el muestreo. El tamaño de la población en 2018 era de 16 estudiantes. El 38\% de ellos ingresa en el año 2015, el 19\% en el 2017 y el $43 \%$ en el 2018.

Se utiliza el cuestionario como instrumento de recolección de datos, según Hernández et al. "consiste en un conjunto de preguntas respecto de una o más variables a medir" (2010, p. 217). Por ello, se emplea para conocer 
la percepción de los estudiantes indígenas del Campus Sarapiquí de la Universidad Nacional (UNA), sobre su inserción y desempeño en los cursos de aplicaciones computacionales de la carrera de Administración de Oficinas, además para identificar cómo los antecedentes culturales y académicos indígenas influyen en su aprendizaje.

El instrumento abarca las variables de la investigación y se conforma por preguntas cerradas y algunas abiertas relacionadas con datos personales de los encuestados, situación: actual sobre la carrera, características de su etnia, formación en secundaria, cursos matriculados en el área de la tecnología, reprobación de materias y sus causas y recomendaciones estudiantiles sobre el desarrollo de los cursos. Los datos obtenidos mediante la aplicación del cuestionario fueron procesados por las investigadoras mediante el programa informático Microsoft Excel. Seguidamente, con base en la información se elaborarán gráficos, tablas, cuadros estadísticos para facilitar el análisis y síntesis de los datos.

Posteriormente, se realiza el análisis e interpretación con el objetivo de conocer la percepción de los estudiantes y sus recomendaciones. Como producto del proceso anterior, se brindan las conclusiones y recomendaciones respectivas.

\section{RESULTADOS}

Con base en el instrumento de recolección de datos utilizado se aplica un cuestionario con preguntas abiertas y cerradas, permitiendo obtener los resultados que a continuación se presentan. Los estudiantes participantes oscilan en un $50 \%$ en el rango de edad de 18 a 22 años; el $31 \%$, en el rango de 23 a 27 años; el 13\%, de 28 a 32 años y un $6 \%$ de 38 a 42 años. Referente al nivel de la carrera que está cursando, al 2018 , el $44 \%$ de ellos se encuentra en I nivel; el $25 \%$, en II nivel; el $6 \%$, en IV nivel y el otro $25 \%$ se encuentra en diferentes niveles, por pérdida de cursos.

En relación con el grupo étnico al que pertenecen, el $63 \%$ es Bribri; el $31 \%$, Cabécar, y el $6 \%$ Maleku. De ellos, el $69 \%$ vive en el territorio indígena de Talamanca Bribri y el $31 \%$ en Talamanca Cabécar.

Se consulta a los estudiantes si hablan alguna lengua indígena, a lo que el $81 \%$ reporta hacerlo. Únicamente el $19 \%$ responde negativamente; 
además, el $63 \%$ utiliza el español como la lengua que mayormente emplea para comunicarse con su familia y amigos; el 19\%, lengua indígena y el $18 \%$ ambos idiomas.

Por otra parte, el $88 \%$ de los estudiantes encuestados recibía sus clases de colegio en español; el $6 \%$, en lengua indígena y el $6 \%$ restante en ambos idiomas, dato que evidencia que el idioma en español no es un problema de aprendizaje en los cursos de tecnología. Asimismo, es importante indicar que el $100 \%$ de los encuestados se graduó de secundaria de un colegio en territorio indígena.

Adicionado a lo anterior, el $81 \%$ tuvo profesores de secundaria indígenas. De este porcentaje, el $66 \%$ indica que fueron en su mayoría indígenas y el $15 \%$ tuvo en su minoría docentes indígenas. Únicamente el $19 \%$ no tuvo profesores indígenas en sus centros de estudio. Esta información indica que sí existe un cambio relacionado a la figura del facilitador en el proceso de enseñanza, aspecto que puede incidir dentro de su integración en el contexto educativo.

Un aspecto muy significativo como parte de esta investigación es que el $75 \%$ de los encuestados no recibió ningún curso sobre tecnologías o manejo de la computadora dentro de su formación secundaria, dato que evidencia la carencia de formación en esta área y el impacto que reciben los estudiantes al ingresar a la Universidad y tener que hacer frente a la utilización eficiente de las tecnologías de la información. Únicamente el $25 \%$ recibió algún curso; no obstante, los estudiantes indican que la clase consistía en jugar, que fue un curso muy básico y, además, que las clases no fueron continuas.

En relación con la reprobación de cursos en el área de tecnología en la carrera Administración de Oficinas, el $44 \%$ ha perdido algún curso: tres estudiantes, la materia de Técnicas Mecanográficas por Computadora I; tres, la materia de Técnicas Mecanográficas por Computadora II y otro, Técnicas Mecanográficas por Computadora III.

En el instrumento aplicado se les brinda una serie de opciones con el fin de que ellos indiquen cuáles, según su percepción, eran las causas originarias para la reprobación de los cursos en el área de tecnología. La tabla 1 muestra los resultados obtenidos. Se evidencia que las principales 
causas obedecen a problemas de aprendizaje, problemas familiares, desadaptación al medio, falta de comprensión de contenidos, falta de hábitos y técnicas de estudio, causas económicas y origen psicológico.

\section{Tabla 1}

Causas de reprobación de cursos en el área de tecnología

\begin{tabular}{lll}
\hline Causa & $\begin{array}{l}\text { Total } \\
\text { Absoluto }\end{array}$ & $\begin{array}{l}\text { Total } \\
\text { Relativo }\end{array}$ \\
\hline $\begin{array}{l}\text { Causas atribuibles a problemas de aprendizaje: } \\
\text { adecuaciones curriculares, dificultad para el uso de la } \\
\text { tecnología }\end{array}$ & 5 & $71 \%$ \\
$\begin{array}{l}\text { Causas de origen familiar } \\
\begin{array}{l}\text { Desadaptación al medio (vida universitaria) por el origen } \\
\text { sociocultural del que provienen }\end{array}\end{array}$ & 4 & $43 \%$ \\
$\begin{array}{l}\text { Falta de comprensión de los contenidos desarrollados } \\
\text { en el curso }\end{array}$ & 3 & $57 \%$ \\
$\begin{array}{l}\text { Falta de hábitos y técnicas de estudio } \\
\text { Causas económicas }\end{array}$ & 2 & $43 \%$ \\
$\begin{array}{l}\text { Causas de origen psicológico } \\
\text { Problemas de salud }\end{array}$ & 2 & $28 \%$ \\
$\begin{array}{l}\text { Desubicación en propósitos de vida e inadecuada } \\
\text { opción vocacional }\end{array}$ & 2 & $28 \%$ \\
$\begin{array}{l}\text { Causas relacionadas con el o la docente: dominio del } \\
\text { tema, estrategias didácticas, evaluación y metodología } \\
\text { utilizada }\end{array}$ & 0 & $28 \%$ \\
\begin{tabular}{l} 
Otras: por favor indique \\
\hline
\end{tabular} & 0 & $0 \%$ \\
\hline
\end{tabular}

Fuente: Elaboración propia, tomado del resultado de la encuesta realizada.

Por otra parte, se consultó a los encuestados qué recomendaciones harían con respecto al desarrollo de los cursos de tecnología, de manera que al estudiante indígena se le facilite el proceso de aprendizaje. Al respeto, los estudiantes manifestaron que la formación que recibieron en sus centros de estudios es muy baja y que, además, en sus territorios de origen las herramientas tecnológicas son muy limitadas, por lo que cuando llegan a la Universidad no tienen conocimientos previos, lo que los pone en desventaja ante sus compañeros de clase. Indican que les gustaría recibir 
charlas o talleres previos a los cursos de carrera para nivelar contenidos en computación. También, manifiestan que es necesario que los contenidos se desarrollen lentamente, que el docente tenga mucha paciencia y, además, que verifique constantemente si los contenidos están quedando claros mediante atención personalizada. Finalmente, indican que el estudiante tiene la responsabilidad de hacer uso de los espacios para evacuar dudas y ampliar temas, como lo es la hora atención al estudiante y además practicar mucho de manera independiente.

Las respuestas emitidas por los estudiantes denotan la carencia de formación en el área de aplicaciones computacionales y la importancia de recibir los cursos de manera más minuciosa y detallada, con explicaciones en lenguajes sencillos, así como brindar cursos niveladores al inicio de la carrera, que les permitan adquirir conocimientos básicos sobre el uso de la computadora.

Complementariamente, para el $100 \%$ de los estudiantes es importante adquirir conocimientos y desarrollar habilidades y competencias para el buen manejo de las aplicaciones tecnológicas y equipos computacionales de uso en la oficina, debido a que para ellos la tecnología está presente en cada aspecto de la vida y les permite ser más eficientes en el trabajo. Para los encuestados, el manejo de la tecnología diversifica sus oportunidades, les permite ser competitivos en el ambiente laboral, les facilitará conseguir trabajo con mayor facilidad y mantenerse vigentes en su trabajo; además, en su condición de indígenas les abre las puertas para socializar y compartir con otros jóvenes de su entorno. De las respuestas anteriores, se desprende que la población indígena asocia el uso de las aplicaciones informáticas con buenas prácticas administrativas que les permitan ser competentes, fomentar su desarrollo personal y lograr una buena adaptación al mercado laboral.

Finalmente, se consulta al estudiantado si ellos consideran que el docente, como parte del desarrollo del curso, debería realizar modificaciones para la inclusión de su cultura, para lo que se obtuvo como resultado que para el $100 \%$ no es necesario. Lo anterior, por cuanto mantener las prácticas de su cultura es su responsabilidad y no esperan que el mundo se adapte a ellos, sino más bien, esperan desarrollar la habilidad de ser competitivos en el mundo laboral en respeto de sus raíces. Además, ellos consideran que no es posible adaptar la tecnología a su cultura pues en esta, la 
computadora no existe; pero son conscientes de que para quienes deciden estudiar, su uso es indispensable, por lo que asumen la responsabilidad de su adaptación.

La información anterior llama la atención de las investigadoras, en cuanto la población indígena es muy clara de la ideología de su cultura. Asimismo, se desprenden recomendaciones en cuanto a generar metodologías más participativas y una mayor comunicación entre el docente y el estudiante. Los jóvenes son enfáticos en mencionar que ellos son los responsables de adaptarse sin perder las prácticas culturales.

\section{DISCUSIÓN Y CONCLUSIONES}

Se determina que es inminente la necesidad de que todas las personas aprendan sobre tecnologías. Esto por cuanto todo lo que se desarrolla a su alrededor está influenciado por ellas: los electrodomésticos que se utilizan en los hogares, los medios de transporte, la comunicación, la ciencia, la medicina, la construcción, las relaciones laborales y, para efectos de la temática que compete a esta investigación, las empresas.

Actualmente, las oficinas gestionan sus procesos y actividades a través de sistemas de información especializados: paquetes computacionales, internet, dispositivos y equipos tecnológicos, lo que obliga a los futuros colaboradores administrativos a tener dominio de las tecnologías de información y comunicación que rigen el mundo de los negocios. De lo anterior se deduce que las carreras con enfoque administrativo que brindan las diferentes universidades incluyan en sus planes de estudio temáticas relacionadas con las tecnologías y su aplicabilidad en las empresas. Tal es el caso de la carrera Administración de Oficinas de la Universidad Nacional, que tiene como una de sus áreas generales de estudio la tecnología y que, a su vez, se divide en siete cursos sobre esta temática.

Para muchos estudiantes, la implementación de la tecnología es parte de su diario vivir, pues están acostumbrados a usar cotidianamente internet, teléfono celular y su computadora portátil. Para ellos, el aprendizaje de aplicaciones computacionales no representa ninguna complicación; más bien, se sienten familiarizados con estas temáticas. Sin embargo, hay poblaciones, como la indígena, que no están acostumbradas a lidiar con tecnologías y para quienes la implementación de estos saberes representa 
todo un reto. Se evidencian desigualdades en las posibilidades que existen para acceder a la información, al conocimiento y a la educación, mediante el uso de la tecnología.

El $44 \%$ de los estudiantes matriculados actualmente ha perdido algún curso del área de tecnología. Sin embargo, la mayoría refiere que sus limitaciones son personales y no las atribuyen a la forma en la que se desarrollan los cursos.

Aunque los estudiantes asumen la responsabilidad de su formación y adaptación, se evidencia que la falta de formación previa en el área de tecnología hace que ellos ingresen rezagados a la universidad poniéndolos en desventaja en relación con sus compañeros. Esto, aunado al proceso de adaptación que tienen que atravesar para ingresar a la universidad, resulta en una cantidad importante de factores que pueden influenciar negativamente su desempeño escolar.

Se afirma la importancia de recibir cursos de nivelación que les ofrezcan una base de conocimiento, de tal manera que cuando ingresen a los cursos de aplicaciones computacionales propiamente de carrera, se encuentren en igualdad o similitud de condiciones que los otros estudiantes. A partir de ahí, y con la colaboración del docente ofreciendo explicaciones en lenguajes sencillos, asignando mucha práctica y propiciando espacios extracurriculares para reforzar y practicar contenidos, es posible que los estudiantes logren mejorar su rendimiento.

Un aspecto relevante es el hecho de que los estudiantes encuestados mencionan que la computadora no forma parte de su cultura. Más bien, es un elemento nuevo y son enfáticos en que es su obligación adaptarse a los cambios que la universidad les plantea sin que este proceso influya en sus prácticas culturales.

El proceso de enseñanza y aprendizaje bajo sistemas interculturales debe desarrollarse bajo metodologías apegadas al respeto hacia la diversidad, generando relaciones simétricas, incluyentes, de manera que se contemple la construcción de la realidad y la visión de desarrollo. Es necesario contextualizar para la selección de los recursos educativos y los medios didácticos adecuados de forma que sean significativos para la consecución del éxito y la calidad del proceso educativo. 
Asimismo, la investigación permite concluir que la población indígena que estudia Administración de Oficinas en el Campus Sarapiquí de la Universidad Nacional percibe que su inserción a los cursos de aplicaciones computaciones implica fuertes demandas de aprendizaje y adaptación en razón de que no se posee una formación previa en el área tecnológica.

El desempeño de la población indígena asociado a términos de aprobación de los cursos de aplicaciones computacionales denota limitaciones para la obtención de resultados satisfactorios, ya que un porcentaje de jóvenes ha reprobado uno o más cursos de la carrera. El aprendizaje de la tecnología en esta población presenta realidades distintas, principalmente por el acceso a los recursos y al conocimiento; existe un rezago evidente en cuanto a la manipulación del computador. No obstante; para todos los estudiantes, formarse y estar a la vanguardia de los cambios tecnológicos es necesario para su desempeño personal y profesional.

En los cursos de aplicaciones computacionales de la carrera Administración de Oficinas del Campus Sarapiquí de la Universidad Nacional, por parte de las investigadoras se han implementado metodologías que permitan el aprendizaje colaborativo y dinámico, haciendo uso de técnicas didácticas que faciliten el desarrollo de destrezas y habilidades en la manipulación de las aplicaciones computacionales de uso frecuente en la oficina. Entre las principales utilizadas se encuentran: visitas y experiencias directas con propósito, discusiones guiadas, utilización de medios audiovisuales, programas de aplicación informáticos, juegos educativos, programas de ejercitación, tutoriales digitales, internet, correo electrónico, foros de discusión, exposición verbal, conversaciones electrónicas, aulas virtuales, prácticas guiadas, trabajo en grupos, trabajo por proyectos y presentaciones en programas informáticos.

Se logra identificar cómo los antecedentes culturales y académicos de los estudiantes indígenas influyen negativamente en su aprendizaje. Por mencionar algunos, que todos tuvieron que trasladar su domicilio para estar cerca de la universidad, desarrollar nuevas relaciones interpersonales, hablar lenguas distintas, contar con recursos tecnológicos limitados en sus comunidades, no estudiar tecnologías en la secundaria, tener una formación previa prácticamente nula, presentar problemas familiares y económicos, problemas de aprendizaje y de origen psicológico, falta de hábitos y 
técnicas de estudio, así como formar parte de una comunidad con distintas costumbres y hábitos.

Una educación intercultural exige un trabajo entre iguales: mismos derechos y condiciones, implica un intercambio de saberes. Debe ser inclusiva y diversa, así como respetuosa y comprensiva. Se construye en conjunto bajo sistemas flexibles y dinámicos.

La Universidad Nacional, a través de sus principios, tiene trazado el camino hacia el conocimiento transformador, el humanismo, la inclusión así como en su valores por medio del compromiso social, la equidad, la participación democrática y el respeto; aspectos que se entrelazan en sus fines reflejados en el diálogo de saberes, la identidad y el compromiso, la formación integral y el pensamiento crítico. Por su parte, la Sección Regional Huetar Norte y Caribe de la UNA, a través de su declaratoria como Campus Intercultural, trabaja con miras a mejorar procesos interculturales.

El papel del docente debe enmarcarse en una actitud de colaboración, pero también de aprendizaje. Es necesario aprender sobre el contexto socio-cultural de la población indígena para trabajar las clases en respeto de sus necesidades $y$, además, dar paso al reconocimiento de aportes y diferencias de otras culturas, permitiendo que la educación superior costarricense se rija por principios de interculturalidad y facilitando así la permanencia de los estudiantes indígenas en la Universidad.

\section{RECOMENDACIONES}

De la recolección de información se desprenden también recomendaciones en cuanto a generar metodologías más participativas y una mayor comunicación entre el docente y el estudiante para facilitar el proceso educativo. Con base en la observación y el análisis de los resultados obtenidos con la encuesta aplicada a la población de interés, se brindan las siguientes:

\section{A los estudiantes indígenas}

- Fomentar las habilidades de comunicación con el fin de informar al profesor sobre sus necesidades y limitantes en el proceso de enseñanza. 
- Establecer buenos hábitos de estudio que les permitan reforzar los conocimientos, las habilidades y las destrezas que se deben adquirir en los cursos de tecnología en la carrera Administración de Oficinas.

- Hacer uso de las facilidades extracurriculares que les ofrece la UNA, Campus Sarapiquí, para sacar el mayor provecho de su formación académica: computadoras disponibles para uso de estudiantes en la biblioteca, hora estudiante (tiempo adicional que ofrece el docente en todos los cursos de aplicaciones computacionales) y, en caso de ser necesario, gestionar tutorías adicionales ante el Programa Éxito Académico para aclarar dudas y practicar las temáticas desarrolladas en los cursos.

- Comunicar, tanto al docente como a la trabajadora social, cualquier situación personal que pueda afectar sus estudios, de manera que se verifique la posibilidad de ofrecer alguna colaboración desde la institución que aminore cualquier impacto negativo que dicha situación pueda tener sobre su educación.

\section{A los profesores que trabajan con población indígena}

- Aplicar un diagnóstico socio-cultural y académico que permita conocer tanto el contexto como los conocimientos previos de los estudiantes, con el fin de tomar en cuenta las necesidades específicas de esta población al desarrollar los cursos de tecnología.

- Recomendar a la administración universitaria que se brinde a la población indígena talleres o cursos introductorios sobre el uso de las principales aplicaciones informáticas, así como actividades de capacitación para los docentes que les brinden las herramientas necesarias para desarrollar procesos de enseñanza y aprendizaje dentro del marco de interculturalidad.

- Organizar con ayuda de la administración universitaria, actividades que den a conocer las prácticas culturales de las poblaciones indígenas que forman parte del Campus.

- Generar espacios que permitan a la población indígena aclarar dudas y desarrollar prácticas de los contenidos desarrollados en el curso. 
- Fomentar las habilidades de comunicación, con el fin de comprender mejor las necesidades y limitantes de la población indígena.

- Desarrollar los cursos de aplicaciones computacionales con términos sencillos y explicaciones detalladas que permitan la comprensión de los contenidos.

- Propiciar la realimentación por parte de los estudiantes en relación con los avances y limitaciones que experimentan durante las lecciones.

\section{REFERENCIAS}

Amado M., García, A., Brito, R., Sánchez, B. y Sagaste, A. (2014). Causas de reprobación en ingeniería desde la perspectiva del académico y administradores. Ciencia y Tecnología, 14, 233-250. Recuperado de: http://www.palermo.edu/ingenieria/pdf2014/14/CyT_14_15.pdf

Barragán, J. y Vela, J. H. (2015). Impacto de las TICs en la Gestión de Negocios Internacionales. Daena: International Journal of Good Conscience, 10(3), 18-33. Recuperado de http://www.spentamexico.org/ v10-n3/A3.10(3)18-33.pdf

Borge, C. (2012). Costa Rica estado de la educación en territorios indígenas. Cuarto Informe: Estado de la Educación en Costa Rica. San José, Programa Estado de la Nación.

Cabrera, I. y Gallardo, T. (2013). Educación intercultural del estudiante universitario: el enfoque de formación humanístico intercultural. Actualidades investigativas en educación, 13(3). doi: 10.15517/AIE. V1313.12049

Czarny, G. (2007). Pasar por la escuela: metáfora que guarda distintas caras para abordar la relación comunidades indígenas y escolaridad. Revista Mexicana de Investigación Educativa, 12(34), 921-950. Recuperado de http://www.redalyc.org/articulo.oa?id=14003406

Consejo Nacional de Rectores [CONARE]. (2013). Proyecto de Mejora de la Educación Superior, Marco de Planificación para Pueblos 
Indígenas (MPPI). Recuperado de http://www.documentos.una. ac.cr/handle/unadocs/3290

Fallas Monge, I. y Trejos Trejos, I. (2015). Educación en la Sociedad de la Información y el Conocimiento. San José, Costa Rica: EUNED.

García, J. (2016). Influencia de las tics en la educación: inconvenientes, uso y aportes al sistema educativo. Revista Electrónica Campus Virtual de la Universidad Fermín Toro, 9(3), 104-125. Recuperado de http://www.uft.edu.ve/campusvirtual/revistacampusvirtual\%20 Edi.\%20IX\%20(9)/edicion\%20IX.pdf

Gay, A. (2008). Tecnología y sociedad. Latin American and Caribbean Journal of Engineering Education, 2(2), 47-50. Recuperado de http://eds.a.ebscohost.com/eds/pdfviewer/pdfviewer?vid=1\&sid=d372fd85-3ed1-43be-b26f-c7883f0cd0a8\%40sessionmgr4008

González Oviedo, M. (2009). Educación e Interculturalidad: lo nuestro, lo propio, lo de todos. San José, Costa Rica: Ministerio de Educación Pública.

Hernández, I. y Calcagno, S. (2003). Los pueblos indígenas y la sociedad de la información. Revista Argentina de Sociología, 1(1). 110-143.

Hernández, R., Fernández, C., y Baptista, P. (2010). Metodología de la Investigación. México: McGraw Hill.

Mandiola E. (s.f.). Educación e Interculturalidad. UNESCO. Recuperado de http://www.unesco.org/new/es/quito/education/ education-and-interculturality/\#topPage

Luján, A. y Torres, J. (2014). Jóvenes indígenas y educación superior. Facultad de Humanidades y Ciencias de la Educación. VIII Jornadas de Sociología de la UNLP (La Plata). Objeto de conferencia llevada a cabo en Chaco, Argentina. Recuperado de http://hdl.handle. net/10915/55919

Paladino, M. (2009). Pueblos Indígenas y educación superior en Argentina. Datos para el debate. ISEES: Inclusión Social y Equidad en la Educación Superior, (06), 81-122. 
Prada Ospina, R. (2016). Las TIC, factor de efectividad en la aplicación del mercadeo digital en negocios de retail. Revista Escuela Administración de Negocios, (80), 105-116. Recuperado de http://www.scielo. org.co/pdf/ean/n80/n80a08.pdf

Prensky, M. (2011). Enseñar a nativos digitales. Estados Unidos: SM Ediciones.

Programa Estado de la Nación (2012). Reconocimiento y exigibilidad de derechos de los pueblos indígenas. Decimoctavo Informe Estado de la Nación en Desarrollo Humano Sostenible. San José, Programa Estado de la Nación.

Saavedra, L. E. (2017). Las TIC en el campo de la gestión administrativa empresarial. Multi-Ensayos, 2(4), 107-112. Recuperado de http://www.revistasnicaragua.net.ni/index.php/multiensayos/article/ view/2977

Salazar, R. (2006). El indígena costarricense: una visión etnográfica. Cartago: Editorial Tecnológica de Costa Rica.

Sánchez, A., Cabraca A., Odio, B., Torres, C., Chacón, D., Pérez, E., (...) y Rivera, Y. (2009). Reconstruyendo el concepto de interculturalidad. Boletín electrónico División de Educación Rural, Universidad Nacional (3). Recuperado de http://www.redrural.una.ac.cr/index.php?option=com_content\&view=article\&id=105:reconstruyendo-el-concepto-de-interculturalidad \&catid=58:vol-3\&ltemid=81

Seas Tencio, J. (2016). Didáctica General I. San José, Costa Rica: EUNED.

Solarte, M. F., Urbano, F. A., Triviño, G. L. (2007). B-Learning para Capacitación de Profesores en Alfabetización Digital: Una Estrategia para el Mejoramiento de la Inclusión Social en el Resguardo Indígena de Guambía. Revista Avances en Sistemas e Informática, 4(3), 65-76. Recuperado de http://www.redalyc.org/articulo.oa?id=133115019009

Viveros-Márquez, J. y Moreno-Olivos, T. (2014). El Enfoque Intercultural Bilingüe y su impacto en la calidad de la educación indígena. Estudio de Caso. Ra Ximhai, 10(3), 55-73. 
